\title{
A Risk Score to Guide Cystatin C Testing to Detect Occult-Reduced Estimated Glomerular Filtration Rate
}

\author{
Carmen A. Peralta Paul Muntner Rebecca Scherzer Suzanne Judd \\ Mary Cushman Michael G. Shlipak \\ San Francisco Veterans Affairs Medical, San Francisco, Calif., USA
}

\section{Key Words}

Kidney disease - Serum cystatin C . Creatinine .

Creatinine-based estimated glomerular filtration rate .

Cystatin C-based estimated glomerular filtration rate

\begin{abstract}
Background/Aims: Persons with occult-reduced estimated glomerular filtration rate (eGFR $<60 \mathrm{ml} / \mathrm{min} / 1.73 \mathrm{~m}^{2}$ detected by serum cystatin $C$ but missed by creatinine) have high risk for complications. Among persons with preserved kidney function by creatinine-based eGFR (eGFRcreat $>60 \mathrm{ml} /$ $\min / 1.73 \mathrm{~m}^{2}$ ), tools to guide cystatin $C$ testing are needed. Methods: We developed a risk score to estimate an individual's probability of reduced eGFR by cystatin C (eGFRcys $<60$ $\mathrm{ml} / \mathrm{min} / 1.73 \mathrm{~m}^{2}$ ) in The Reasons for Geographic and Racial Differences in Stroke (REGARDS) study and externally validated in the Third National Health and Nutrition Examination Survey (NHANES III). We used logistic regression with Bayesian model averaging and variables available in practice. We assessed performance characteristics using calibration and discrimination measures. Results: Among 24,877 adults with preserved kidney function by creatinine, $13.5 \%$ had reduced eGFRcys. Older and Black participants, current
\end{abstract}

smokers and those with higher body mass index, lower eGFRcreat, diabetes, hypertension and history of cardiovascular disease were more likely to have occult-reduced eGFR $(p<0.001)$. The final risk function had a c-statistic of 0.87 in REGARDS and 0.84 in NHANES. By risk score, $72 \%$ of occultreduced eGFR cases were detected by screening only $22 \%$ of participants. Conclusions: A risk score using characteristics readily accessible in clinical practice can identify the majority of persons with reduced eGFRcys, which is missed by creatinine.

(c) 2015 S. Karger AG, Basel

\section{Introduction}

Reduced estimated glomerular filtration rate (eGFR), defined as $<60 \mathrm{ml} / \mathrm{min} / 1.73 \mathrm{~m}^{2}$, is associated with increased risks of cardiovascular events, death and progression to end-stage renal disease (ESRD) [1]. Accurate detection and classification of persons at highest risk for complications from reduced eGFR is required in order to implement targeted prevention strategies. In fact, international guidelines now recommend that diagnosis and staging of chronic kidney disease (CKD) should be based

\section{KARGER 125}

(c) 2015 S. Karger AG, Base

$0250-8095 / 15 / 0422-0141 \$ 39.50 / 0$

E-Mail karger@karger.com

www.karger.com/ajn
Carmen A. Peralta, MD, MAS

4150 Clement Street

111A1, San Francisco, CA 94121 (USA)

E-Mail carmenalicia.peralta@ucsf.edu 
on categories of both creatinine-based eGFR (eGFRcreat) and urinary albumin levels to reflect disease prognosis [2]. However, reliance on eGFRcreat alone to detect reduced eGFR can misclassify the risk associated with CKD. In a meta-analysis of 11 general population cohorts with over 90,000 participants, more than $14 \%$ of people with eGFRcreat $>60 \mathrm{ml} / \mathrm{min} / 1.73 \mathrm{~m}^{2}$ were reclassified to an eGFR $<60 \mathrm{ml} / \mathrm{min} / 1.73 \mathrm{~m}^{2}$ by cystatin C. These persons were at significantly higher risks for death, cardiovascular death and ESRD, compared to those who were not reclassified [3].

The most recent Clinical Practice Guideline for the Evaluation and Management of Chronic Kidney Disease included a new suggestion to confirm CKD by serum cystatin C-based eGFR (eGFRcys) among persons with eGFRcreat of $45-59 \mathrm{ml} / \mathrm{min} / 1.73 \mathrm{~m}^{2}$ and no albuminuria, particularly if the clinician suspects the eGFRcreat may be inaccurate. However, the guideline provided little guidance on the use of cystatin $\mathrm{C}$ for those with higher eGFRcreat levels. In addition to improved classification of persons who had eGFRcreat of $45-59 \mathrm{ml} / \mathrm{min} / 1.73 \mathrm{~m}^{2}$, we have previously shown that measurement of cystatin $\mathrm{C}$ can capture 'occult-reduced eGFR', defined as eGFRcys $<60 \mathrm{ml} / \mathrm{min} / 1.73 \mathrm{~m}^{2}$ among persons with eGFRcreat $>60 \mathrm{ml} / \mathrm{min} / 1.73 \mathrm{~m}^{2}[4,5]$. Despite advances in the field, no systematic approaches have been published to identify persons who are most likely to have reduced eGFR that is missed by creatinine. As cystatin $\mathrm{C}$ remains relatively more expensive to measure compared with creatinine, guidance is needed to identify persons at high risk for occult-reduced eGFR using easily ascertainable clinical and demographic characteristics.

We designed this study to develop and validate a risk score for occult-reduced eGFR in the large, general population of US adults participating in the Reasons for Geographic and Racial Differences in Stroke (REGARDS) study and the National Health and Nutrition Examination Survey (NHANES).

\section{Methods}

\section{Participants}

To develop a risk score for occult-reduced eGFR, we used data from the REGARDS study. REGARDS is a large, populationbased cohort study designed to study factors that contribute to the excess stroke burden among American Blacks and among those in the 'stroke belt' of the United States. Briefly, between 2003 and 2007, REGARDS recruited 30,239 Black and White participants who were 45 years or older. Participants were randomly sampled and recruited by mail and then telephone, followed by an in-home study visit. By design, approximately $50 \%$ of REGARDS participants were recruited in North Carolina, South Carolina, Georgia, Tennessee, Mississippi, Alabama, Louisiana and Arkansas. The other 50\% was recruited from the remaining 40 continental states and Washington, DC. Participant information was first collected via a telephone interview. A trained technician then conducted an in-home examination for the anthropometric and clinical examination, specimen collection and inventory of medications [6].

For these analyses, we included REGARDS participants who had eGFRcreat $>60 \mathrm{ml} / \mathrm{min} / 1.73 \mathrm{~m}^{2}$ and who had a measurement of cystatin C, for a total sample size of 24,877 . All appropriate institutional review boards approved this study, and participants provided written informed consent.

\section{Outcome}

The primary study outcome of interest was occult-reduced eGFR. This was defined as having an eGFRcys $<60$ and eGFRcreat $>60 \mathrm{ml} / \mathrm{min} / 1.73 \mathrm{~m}^{2}$. Cystatin $\mathrm{C}$ was measured by particle-enhanced immunonephelometry (N Latex Cystatin C on the BNII, Dade Behring) after a 12-hour fast and calibrated to the international standard [7]. Serum creatinine was measured and calibrated to isotope dilution mass spectrometry traceable methods. The eGFRcreat was estimated using the CKD-EPI equation for creatinine (eGFRcreat), and the eGFRcys using the 2012 CKD-EPI cystatin $\mathrm{C}$ equation [8].

\section{Candidate Variables}

For these analyses, we considered candidate variables defined a priori as being associated with CKD and likely to be readily available in clinical practice. Age, race, sex and smoking history were determined by self-report during the telephone interview. Height and weight were obtained by a trained technician. Prevalent cardiovascular disease (CVD) was defined by one of the following: electrocardiographic evidence of a myocardial infarction, self-report of a cardiac procedure (CABG or angioplasty), self-reported myocardial infarction or self-reported stroke. Hypertension was defined by self-reported use of antihypertensive medications or an average of 2 seated blood pressure (BP) measurements with systolic BP $\geq 140 \mathrm{~mm} \mathrm{Hg}$ or diastolic BP $\geq 90 \mathrm{~mm} \mathrm{Hg}$. Diabetes was defined as self-reported use of insulin or oral hypoglycemic agents, fasting blood glucose $\geq 126 \mathrm{mg} / \mathrm{dl}$ or a non-fasting blood glucose $\geq 200 \mathrm{mg} / \mathrm{dl}$. A urine albumin to creatinine ratio (ACR) was entered into the model only in a sensitivity analysis described below. For these, the urine albumin was measured by nephelometry using the BNII ProSpec nephelometer (Dade-Behring) and urine creatinine by the Jaffe method using the Modular-P chemistry analyzer (Roche/Hitachi). We defined albuminuria as spot ACR of $\geq 30$ $\mathrm{mg} / \mathrm{g}$.

\section{Risk Score Development and Evaluation}

We first compared demographic and clinical characteristics of REGARDS participants stratified by eGFRcreat category using the Mann-Whitney U test for continuous variables and the Fisher's exact test for categorical variables. To develop the occult-reduced eGFR risk score, we considered the following clinical characteristics as candidate variables: age (continuous), gender, race, body mass index (BMI) (continuous), diabetes, systolic and diastolic BP (continuous), hypertension, use of antihypertensive medication, history of CVD, smoking and eGFRcreat (continuous). We con- 
sidered ACR (continuous) only in a sensitivity analysis, as this may not be readily available in practice, particularly among persons without diabetes. We assessed linearity for continuous predictors adding quadratic terms to the models. We evaluated unadjusted generalized additive models to construct smoothing splines in order to examine the relationships of continuous parameters with the outcome [9]. We used linear splines to model age, eGFRcreat and BMI due to the curvilinear associations of these variables with occult-reduced eGFR. Specifically, the effect of eGFRcreat was modeled linearly but with different slopes in the ranges of 60-75 and $>75 \mathrm{ml} / \mathrm{min} / 1.73 \mathrm{~m}^{2}$, based on observed associations, and the effect of BMI had different slopes at values greater than or less than $40 \mathrm{~kg} / \mathrm{m}^{2}$.

We employed multivariable logistic regression models to evaluate characteristics associated with reduced eGFRcys. We first used stepwise backward selection with a significance level of $\alpha=$ 0.05 to remove candidate covariates. We utilized Bayesian model averaging as an alternative model building approach; predictors with posterior probabilities $>35 \%$ were retained in the model [10]. Models constructed using the 2 approaches were very similar. We used 10-fold cross-validation to compare candidate models and identify the best fitting model.

Using the final model, we developed a point-based risk score for the presence of occult-reduced eGFR, using methods established by the Framingham Heart Study [11]. The total number of points was calculated for each participant using this risk score and associated with the probability of having occult-reduced eGFR. We used c-statistics to assess model discrimination and goodnessof-fit testing to assess calibration. Bootstrap simulation was used to assess over optimism. We assessed model performance by comparing the observed event rates within each decile of probability to expected rates, which represent the predicted rate based on the proportion at risk within each subgroup. These are plotted across the distribution of the risk score for ease of interpretation. We also evaluated the diagnostic performance of discrete risk score thresholds to detect occult-reduced eGFR in the population. We plotted the cumulative probability and the cumulative proportion of eGFRcys $<60 \mathrm{ml} / \mathrm{min} / 1.73 \mathrm{~m}^{2}$ across the distribution of the risk score, separately. We also present the proportion of occult-reduced eGFR cases detected (number of cases detected/total number of cases) and proportion of the population tested at several values of the risk score.

We conducted 2 sensitivity analyses. In the first analysis, we considered the outcome eGFRcys $<60 \mathrm{ml} / \mathrm{min} / 1.73 \mathrm{~m}^{2}$ or ACR $\geq 30 \mathrm{mg} / \mathrm{g}$, as this is the definition of CKD. In the second analysis, we included an additional 2,053 REGARDS participants with eGFRcreat $45-59 \mathrm{ml} / \mathrm{min} / 1.73 \mathrm{~m}^{2}$, and we considered the outcome eGFRcys $<45 \mathrm{ml} / \mathrm{min} / 1.73 \mathrm{~m}^{2}$ due to the known improvement in reclassification by cystatin $\mathrm{C}$ across that eGFRcreat range.

\section{External Validation}

We validated the risk score from REGARDS using data from the NHANES III of 1988-1994. In this analysis, we included 3,908 persons of age $>45$ years who had measured cystatin $C$ and had eGFRcreat $>60 \mathrm{ml} / \mathrm{min} / 1.73 \mathrm{~m}^{2}$. Less than $1 \%$ of study participants were excluded due to missing covariates. We used SAS procedure survey logistics to produce point estimates and standard errors incorporating sampling weights to account for the complex survey sampling design.

Risk Score for Occult-Reduced eGFR
All analyses were conducted using Stata version 11 and SAS version 9.3. Bayesian model averaging was performed using the BMA package for the $\mathrm{R}$ statistical computing language.

\section{Results}

\section{Participant Characteristics}

Among 24,877 REGARDS participants with an eGFRcreat $>60 \mathrm{ml} / \mathrm{min} / 1.73 \mathrm{~m}^{2}$, approximately 19,30 and $51 \%$ had eGFRcreat of $60-75,75-90$ and $>90 \mathrm{ml} /$ $\mathrm{min} / 1.73 \mathrm{~m}^{2}$, respectively. The median age in the overall sample was 63 (interquartile range 57-70) years, of whom $54 \%$ were female and $41 \%$ were Black. There was a high prevalence of obesity in this cohort, with $32 \%$ having BMI of $30-39.9$ and $5.9 \%$ with $\mathrm{BMI} \geq 40$. Participants in the lower eGFRcreat categories were older, and hypertension and CVD were most prevalent among those with the lowest eGFRcreat (table 1).

\section{Characteristics Associated with Occult-Reduced eGFR}

Overall, 3,354 (13.5\%) persons had occult-reduced eGFR. We found that older age, diabetes, hypertension, history of CVD and current smoking were associated with a higher prevalence of eGFRcys $<60 \mathrm{ml} / \mathrm{min} /$ $1.73 \mathrm{~m}^{2}$. A higher BMI was associated with higher prevalence for occult-reduced eGFR, but the slope appeared to be steeper at levels of BMI $>40 \mathrm{~kg} / \mathrm{m}^{2}$ (test for nonlinearity $\mathrm{p}=0.0007$ ). For example, among persons with BMI $<40 \mathrm{~kg} / \mathrm{m}^{2}$, each unit increase of BMI was associated with a $9 \%$ increased odds of occult-reduced eGFR. Among persons with BMI $>40 \mathrm{~kg} / \mathrm{m}^{2}$, each unit increase in BMI was associated with a $16 \%$ increased odds of occult-reduced eGFR. Age and eGFRcreat showed stronger associations with eGFRcys $<60 \mathrm{ml} / \mathrm{min} / 1.73 \mathrm{~m}^{2}$ at higher and lower levels, respectively (test for nonlinearity $\mathrm{p}=0.006$ and $\mathrm{p}<0.0001$ ), with inflection points around age of 70 years and eGFRcreat $75 \mathrm{ml} / \mathrm{min} /$ $1.73 \mathrm{~m}^{2}$. We detected a statistically significant interaction with race and smoking, where associations appeared stronger in Whites. We therefore allowed estimates to vary by race. While we also detected statistically significant $(\mathrm{p}<0.05)$ interactions with race for age, BMI at $<40 \mathrm{~kg} / \mathrm{m}^{2}$ and eGFRcreat at $>75 \mathrm{ml} /$ $\mathrm{min} / 1.73 \mathrm{~m}^{2}$, the estimates were only minimally different between the Blacks and Whites. Including linear splines with race-specific slopes to model the associations of age, BMI $>40 \mathrm{~kg} / \mathrm{m}^{2}$ and eGFRcreat $>75 \mathrm{ml} /$ $\mathrm{min} / 1.73 \mathrm{~m}^{2}$ did not significantly change model performance. Therefore, we removed these interactions from 
Table 1. Characteristics of REGARDS participants by eGFRcreat category

\begin{tabular}{|c|c|c|c|c|}
\hline \multirow[t]{2}{*}{ Parameter } & \multicolumn{3}{|c|}{ eGFRcreat, $\mathrm{ml} / \mathrm{min} / 1.73 \mathrm{~m}^{2}$} & \multirow[t]{2}{*}{$\mathrm{p}$ value } \\
\hline & $60-75(\mathrm{n}=4,637)$ & $75-90(\mathrm{n}=7,599)$ & $>90(\mathrm{n}=12,641)$ & \\
\hline Age, years* & $69(62-76)$ & $67(60-73)$ & $60(55-65)$ & $<0.0001$ \\
\hline$<60$ & $703(15)$ & $1,836(24)$ & $6,001(47)$ & \\
\hline $60-70$ & $1,813(39)$ & $3,195(42)$ & $5,404(43)$ & \\
\hline $70-80$ & $1,641(35)$ & $2,169(29)$ & $1,111(9)$ & \\
\hline$>80$ & $480(10)$ & $399(5)$ & $125(1)$ & \\
\hline Female & $2,372(51)$ & $3,775(50)$ & $7,404(59)$ & $<0.0001$ \\
\hline Black & $1,579(34)$ & $2,464(32)$ & $6,039(48)$ & $<0.0001$ \\
\hline Cigarette smoking & & & & $<0.0001$ \\
\hline Current & $505(11)$ & $871(11)$ & $2,360(19)$ & \\
\hline Past & $1,974(43)$ & $3,212(42)$ & $4,704(37)$ & \\
\hline Never & $2,135(46)$ & $3,496(46)$ & $5,524(44)$ & \\
\hline Diabetes mellitus & $943(20)$ & $1,245(16)$ & $2,593(21)$ & $<0.0001$ \\
\hline CVD & $1,073(24)$ & $1,313(18)$ & $1,526(12)$ & $<0.0001$ \\
\hline Hypertension & $3,012(65)$ & $4,212(56)$ & $6,732(53)$ & $<0.0001$ \\
\hline Systolic BP, mm Hg & $127(118-138)$ & $125(118-137)$ & $125(117-137)$ & $<0.0001$ \\
\hline Diastolic BP, $\mathrm{mm} \mathrm{Hg}$ & $77(70-81)$ & $77(70-81)$ & $78(71-82)$ & $<0.0001$ \\
\hline BMI, kg/m² & $28(25-32)$ & $28(25-32)$ & $29(25-33)$ & $<0.0001$ \\
\hline ACR, mg/g & $7.5(4.5-17.3)$ & $6.8(4.4-13.6)$ & $7.1(4.7-13.2)$ & $<0.0001$ \\
\hline ACR $\geq 30$ mg/g & $724(16)$ & $861(12)$ & $1,386(11)$ & $<0.0001$ \\
\hline
\end{tabular}

the model for ease of interpretation (online suppl. appendix table 1; for all online suppl. material, see www. karger.com/doi/10.1159/000439231).

\section{Risk Score}

In table 2, we present the variables included in the risk score and the points calculated for each characteristic. The range of possible risk scores was 0 (lowest probability) to 32 (highest probability). The observed percentage of participants with occult-reduced eGFR ranged from $<1 \%$ in the lowest decile to $55 \%$ in the highest decile, while the expected probability ranged from $<1$ to $54.8 \%$. Our risk prediction model had good discrimination (overall c-statistic 0.87 ) and calibration, and bootstrap simulation indicated a very low degree of over optimism (bias = 0.0003 ). In a sensitivity analyses we considered albuminuria in 2 ways. First, we added ACR to the model, and this did not change model performance. Each doubling of ACR was associated with a $20 \%$ increased odds of occult-reduced eGFR (OR 1.20 (1.17-1.23), p < 0.0001). Model discrimination was similar (c-statistic 0.87, 95\% CI $0.866-0.879)$. We also modeled the alternative outcome eGFRcys $<60 \mathrm{ml} / \mathrm{min} / 1.73 \mathrm{~m}^{2}$ or ACR $\geq 30 \mathrm{mg} / \mathrm{g}$. This model also performed well and had a c-statistic 0.78 (95\% bias-corrected CI 0.77-0.78).

We were then interested in understanding the value of using the risk score to guide cystatin $\mathrm{C}$ testing for an in-
Table 2. Point-based calculation of occult-reduced eGFR risk score

\begin{tabular}{lcc}
\hline Risk factor & Level & Points \\
\hline Age, years & $45-49$ & 0 \\
& $50-59$ & 1 \\
& $60-69$ & 3 \\
& $70-79$ & 6 \\
& $80-89$ & 9 \\
& $90-98$ & 11 \\
\hline eGFRcreat (per $1 \mathrm{ml} / \mathrm{min} / 1.73 \mathrm{~m}^{2}$ ) & $60-69$ & 14 \\
& $70-74$ & 12 \\
& $75-89$ & 8 \\
& $90-99$ & 4 \\
& $100-110$ & 2 \\
\hline Diabetes & $>110$ & 0 \\
\hline Hypertension & Yes & 1 \\
\hline CVD & Yes & 1 \\
\hline BMI & Yes & 1 \\
\hline Current smoking & $<22$ & 0 \\
& $22-29$ & 1 \\
& $30-39$ & 3 \\
& $40+$ & 8 \\
\hline
\end{tabular}

Calculated by adding points for each characteristic on the left. Possible range of scores $0-39$, observed range of scores $0-32$.

$144 \quad$ Am J Nephrol 2015;42:141-147
DOI: $10.1159 / 000439231$

Peralta/Muntner/Scherzer/Judd/ Cushman/Shlipak 
Fig. 1. Relationship between a number of points in risk score and probability of eGFRcys $<60 \mathrm{ml} / \mathrm{min} / 1.73 \mathrm{~m}^{2} . \downarrow=$ The risk score calculation for a hypothetical 55 year old black man with a eGFRcreat $74 \mathrm{ml} /$ $\mathrm{min} / 1.73 \mathrm{~m}^{2}$, a BMI of $35 \mathrm{~kg} / \mathrm{m}^{2}$, history of hypertension and current smoking would result in a total score of 19 , corresponding to a $31 \%$ probability of having reduced eGFRcys.

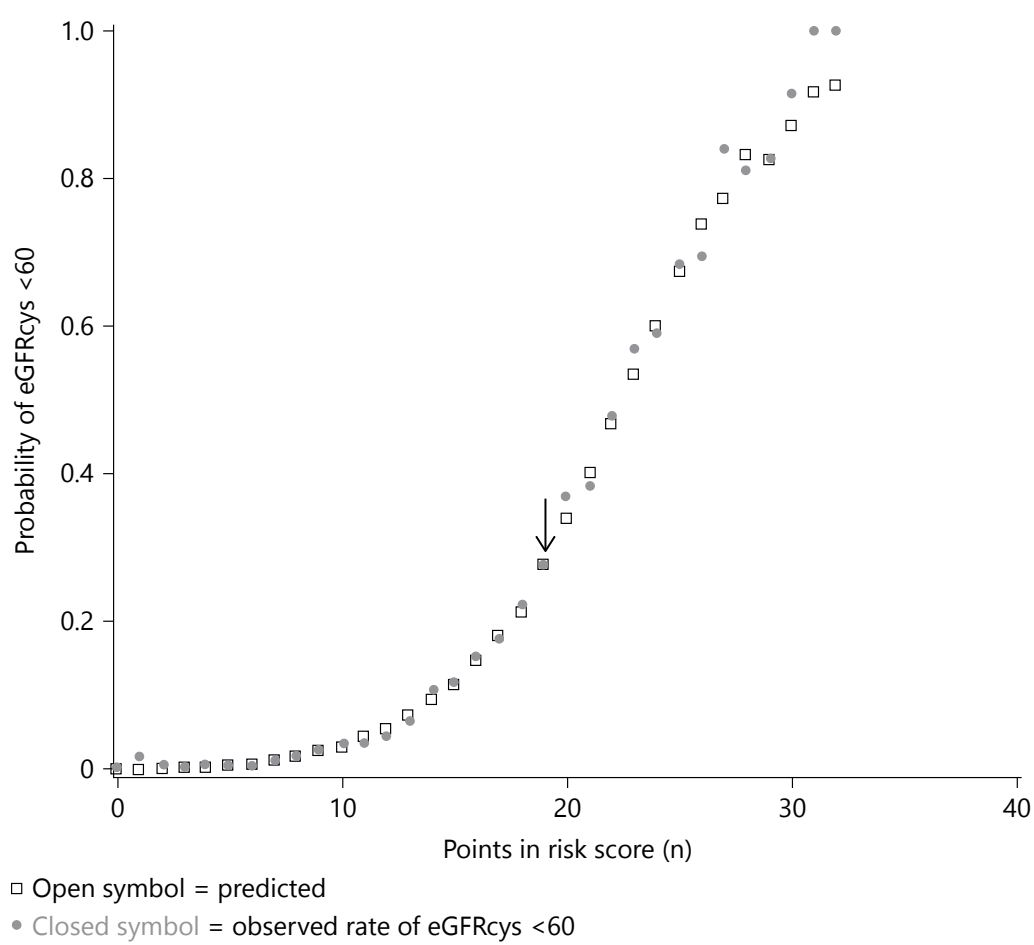

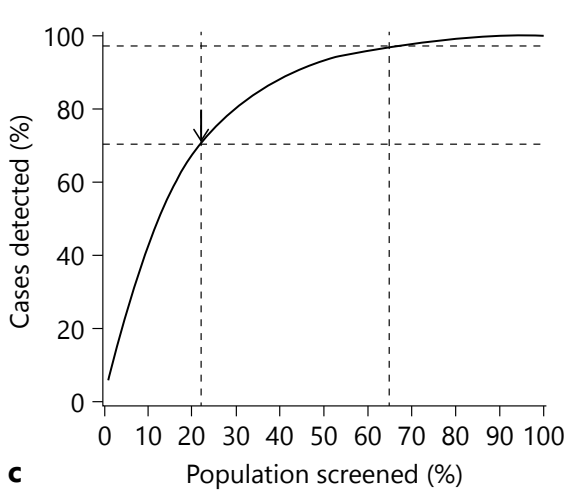

Fig. 2. a-c Value of discrete thresholds of the occult-reduced eGFR risk score. We present an example of how to evaluate the performance of a discrete threshold of the risk score in a population. If we assume that, based on clinical judgment, a clinician would want to test those who have a probability of occult-reduced eGFR $\geq 20 \%$, this threshold corresponds to the 78th percentile of risk (a). Among those above the 78th percentile of risk, we would expect $42 \%$ to have eGFRcys $<60 \mathrm{ml} / \mathrm{min} / 1.73 \mathrm{~m}^{2}(\mathbf{b})$. Such a strategy of testing $22 \%$ of the population would detect $72 \%$ of cases of eGFRcys $<60 \mathrm{ml} / \mathrm{min} / 1.73 \mathrm{~m}^{2}$ (c).

example, if we assume that, based on clinical judgment, a clinician would want to test persons who have a probability of occult-reduced eGFR $\geq 20 \%$, this threshold corresponds to the 78th percentile of risk (fig. 2a). Among those above the 78th percentile of risk, we would expect 
$42 \%$ to have eGFRcys $<60$ (fig. 2 b). Such a strategy of testing $22 \%$ of the population would detect $72 \%$ of cases of eGFRcys $<60$ (fig. 2c).

In a sensitivity analysis including additional participants with eGFRcreat 45-59, we found that the overall probability of eGFRcys $<45$ was $5.3 \%$. The model was well calibrated, and the probability of eGFRcys $<45$ rose with increasing risk score (c-statistic was $0.9339,95 \%$ bias-corrected CI 0.9272-0.9394). Among persons with a risk score $\geq 80$ th percentile, the probability of eGFRcys $<45$ was $4 \%$, and it was $14 \%$ among persons $\geq 90$ th percentile. Approximately $36 \%$ of persons $\geq 90$ th percentile would have eGFRcys $<45$. Only $12 \%$ of the population would be expected to have $\geq 10 \%$ probability of eGFRcys $<45$.

\section{External Validation}

Among participants in NHANES, the survey-adjusted prevalence of occult-reduced eGFR was $12.5 \%$. The model developed in REGARDS had excellent discrimination, with a c-statistic of 0.84 . Associations of each characteristic with occult-reduced eGFR in NHANES are presented in online supplementary appendix table 2 . Online supplementary appendix figure 1 shows a progressive increase in both observed and expected probabilities with increasing decile, which were of similar magnitude, showing good calibration.

\section{Discussion}

In this large cohort of Black and White adults in the United States, we found that characteristics easily ascertained in clinical practice are strongly associated with occult-reduced eGFR. We developed a risk score for occultreduced eGFR using age, race, eGFRcreat, diabetes, BMI, history of CVD, hypertension and current smoking. This risk score had excellent discrimination and calibration, and it performed very well in an external, nationally representative sample. Moreover, using the risk score to guide cystatin $\mathrm{C}$ testing to detect occult-reduced eGFR results in a more efficient use of cystatin $\mathrm{C}$ screening to detect $>70 \%$ of cases, compared to testing strategies using eGFRcreat cut points alone.

Our report expands on the recent KDIGO guideline suggestion to measure cystatin $\mathrm{C}$ for $\mathrm{CKD}$ confirmation among persons with eGFRcreat $45-59 \mathrm{ml} / \mathrm{min} / 1.73 \mathrm{~m}^{2}$ and no albuminuria. The guideline panel cited evidence showing that reliance on eGFRcreat alone can misclassify CKD associated risks in large segments of the popu- lation $[4,5,12]$. Our analyses expand on this suggestion, and add information necessary to evaluate the use of cystatin C among persons with eGFRcreat $>60 \mathrm{ml} /$ $\min / 1.73 \mathrm{~m}^{2}$. Our previous work has shown that occultreduced eGFR is highly prevalent and that those identified as having CKD by eGFRcys, but missed by creatinine, are at high risk for cardiovascular events, death and progression to ESRD $[3,4]$. To our knowledge, this is the first rigorously derived algorithm developed to quantify an individual's probability of having occult-reduced eGFR among persons with preserved eGFR by creatinine.

The risk score presented here has several potential implications. As momentum grows for the use of personalized medicine, a clinician may use the risk score to guide the decision on whether or not to order a cystatin C measurement. The value of detecting occult-reduced eGFR could be for advising patients on their risk for contrast nephropathy, use of NSAIDs or dosing of chemotherapy. Moreover, the recent guidelines from the Eighth Joint National Commission on Hypertension recommend a different $\mathrm{BP}$ threshold in the presence of CKD among persons of age $>60$ years, and first choice agents may differ for persons with CKD [13]. The risk score may also have important potential uses in research. For example, investigators may use the risk score to screen high risk subjects for potential inclusion in studies of persons with $\mathrm{CKD}$, who may otherwise have been excluded. The risk score may also be used in future studies of CKD screening strategies.

A major strength of our study is the large number of participants, the inclusion of Black and White adults across the US, and the parsimonious model which makes it easy to use. We are limited by the lack of evaluation in other ethnic groups, such as Hispanics and Asians, who are also at increased risk for CKD complications. We are limited by our inability to validate the risk score among persons with little muscle mass, such as liver disease or severe cachexia. We are unable to determine the specific contributions of non-GFR determinants of creatinine and cystatin $C$ levels because we do not have a direct GFR measure in this study. This is particularly noteworthy among persons with a high BMI because cystatin C levels may be influenced by fat and inflammation [14], while creatinine is associated with muscle mass [15]. However, direct GFR measurement is not readily available in practice, and clinicians are primarily interested in identifying persons at high risk for CKD complications. Therefore, one should use caution when applying the risk score among persons
Peralta/Muntner/Scherzer/Judd/ Cushman/Shlipak 
with very low or very high BMI, and confirmatory tests are likely needed to diagnose CKD. While the model performed very well in NHANES, testing in these and other groups may still be required. Our risk score does not include estimates of albuminuria. These may not be readily available in practice among persons without diabetes, and different models would be required to guide clinicians on ACR testing. Finally, the cost-effectiveness of the strategies presented here need to be evaluated in future studies.

In summary, we developed a user-friendly algorithm that estimates an individual's probability of having reduced eGFR that is missed by creatinine but detected by cystatin C. Accurate classification of CKD is important in targeting prevention and treatment strategies to persons at highest risk for complications. Future studies to evaluate the cost-effectiveness of measuring cystatin $\mathrm{C}$ guided by this risk score are needed.

\section{Disclosure Statement}

The authors have no conflicts of interest to disclose.

\section{Funding}

C.A.P. is supported by the National Institutes of Diabetes and Digestive and Kidney Diseases (1R03DK095877-01) and a Robert Wood Johnson Harold Amos Award (68519). P.M., S.J. and M.C. are funded by the National Institutes of Health (U01 NS41588). R.S. and M.G.S. are supported by NIH/NIDDK(1R03DK095877-01; Peralta).

\section{References}

1 Go AS, Chertow GM, Fan D, McCulloch CE, Hsu CY: Chronic kidney disease and the risks of death, cardiovascular events, and hospitalization. N Engl J Med 2004;351: 1296-1305.

2 KDIGO: KDIGO 2012 clinical practice guideline for the evaluation and management of chronic kidney disease. Kidney Int Suppl 2013;3:1-150.

-3 Shlipak MG, Matsushita K, Ärnlöv J, et al: Cystatin $\mathrm{C}$ versus creatinine in determining risk based on kidney function. N Engl J Med 2013;369:932-943.

4 Peralta CA, Shlipak MG, Judd S, et al: Detection of chronic kidney disease with creatinine, cystatin $\mathrm{C}$, and urine albumin-to-creatinine ratio and association with progression to endstage renal disease and mortality. JAMA 2011; 305:1545-1552.

$>5$ Peralta CA, Weekley CC, Li Y, Shlipak MG: Occult chronic kidney disease among persons with hypertension in the United States: data from the National Health and Nutrition Surveys 1988-1994 and 1999-2002. J Hypertens 2013;31:1196-1202.

6 Howard VJ, Cushman M, Pulley L, et al: The reasons for geographic and racial differences in stroke study: objectives and design. Neuroepidemiology 2005;25:135-143.

7 Shlipak MG, Sarnak M, Katz R, et al: Cystatin$\mathrm{C}$ and risk for mortality and cardiovascular disease in elderly adults. N Engl J Med 2005; 352:2049-2060.

8 Inker LA, Schmid CH, Tighiouart H, et al: Estimating glomerular filtration rate from serum creatinine and cystatin C. N Engl J Med 2012;367:20-29.

9 Hastie T, Tibshirani R: Generalized Additive Models. CRC Press, 1984.

-10 Hoeting JA, Madigan D, Raftery AE, Volinsky CT: Bayesian model averaging: a tutorial. Stat Sci 1999; 14:382-417.

11 Sullivan LM, Massaro JM, D’Agostino RB Sr: Presentation of multivariate data for clinical use: the Framingham study risk score functions. Stat Med 2004;23:1631-1660.

-12 Peralta CA, Katz R, Sarnak MJ, et al: Cystatin $\mathrm{C}$ identifies chronic kidney disease patients at higher risk for complications. J Am Soc Nephrol 2011;22:147-155.

13 James PA, Oparil S, Carter B, et al: Evidencebased guideline for the management of high blood pressure in adults: report from the panel members appointed to the eighth Joint $\mathrm{Na}$ tional Committee (JNC 8). JAMA 2014;311: 507-520.

14 Knight EL, Verhave JC, Spiegelman D, et al: Factors influencing serum cystatin $\mathrm{C}$ levels other than renal function and the impact on renal function measurement. Kidney Int 2004;65:1416-1421.

15 Chew-Harris JS, Florkowski CM, George PM, Elmslie JL, Endre ZH: The relative effects of fat versus muscle mass on cystatin $\mathrm{C}$ and estimates of renal function in healthy young men. Ann Clin Biochem 2013;50:39-46. 